\title{
« La magie des voix dans la nuit »: « transcréation » des contes de Perrault chez Angela Carter
}

\section{Martine Hennard Dutheil de la Rochère}

\section{Q OpenEdition}

1 Journals

Édition électronique

URL : http://journals.openedition.org/edl/1234

DOI : $10.4000 /$ edl. 1234

ISSN : 2296-5084

Éditeur

Université de Lausanne

\section{Édition imprimée}

Date de publication : 15 septembre 2016

Pagination : 87-108

ISBN : 978-2-940331-49-9

ISSN : 0014-2026

Référence électronique

Martine Hennard Dutheil de la Rochère, « « La magie des voix dans la nuit » : « transcréation » des contes de Perrault chez Angela Carter », Études de lettres [En ligne], 3 | 2016, mis en ligne le 15

septembre 2019, consulté le 15 décembre 2020. URL : http://journals.openedition.org/edl/1234; DOI : https://doi.org/10.4000/edl.1234

\section{(c) Études de lettres}




\section{"LA MAGIE DES VOIX DANS LA NUIT»: "TRANSCRÉATION" DES CONTES DE PERRAULT CHEZ ANGELA CARTER}

Cet article propose une lecture comparative des enjeux de l'oralité dans les contes de Perrault et leurs «reformulations» radiophoniques par Angela Carter (1940-1992) dans le but d'éclairer la démarche de "transcréation" propre à l'auteure féministe britannique qui endosse avec humour le rôle d'une nouvelle «ma mère l'Oye». A partir de sa traduction des contes de Perrault pour les enfants dans The Fairy Tales of Charles Perrault (1977), Carter a exploré la textualité et l'intertextualité des contes dans The Bloody Chamber (1979), un recueil de réécritures destinées à des lecteurs adultes. En contrepoint de ces nouvelles en prose, elle a mis en évidence la dimension orale des contes pour la radio, puis leur dimension visuelle au cinéma. Chez Carter, le conte devient un objet d'expérimentation poétique, générique et médiatique au service de l'émancipation des femmes et de leur contribution à l'histoire culturelle. L'exemple développé ici est celui de Vampirella (1976), un "radio play" qui inspirera aussi à l'auteure une nouvelle intitulée "The Lady of the House of Love». Vampirella revisite "La Belle au Bois dormant» sur le mode gothico-parodique afin de recréer pour ses auditeurs l'expérience archaïque du conte raconté au coin du feu et «la magie des voix dans la nuit».

Open any page and a full score rises from its word-notes, of winds howling, teardrops falling, diamond earrings tinkling, snapping teeth, sneezing, and wheezing. Storytelling for Angela Carter was an island full of noises and sweet airs, and like Caliban, who heard a thousand twangling instruments hum about his ears, she was tuned to an ethereal universe packed with sensations, to which she was alive with every organ.

M. Warner, "Marina Warner on why Angela Carter's The Bloody Chamber Still Bites". 
Le propre du conte est de se réinventer dans le jeu sans fin de la répétition et de la différence. Tout l'art de raconter une histoire consiste à raviver une mémoire collective au gré de l'inspiration, des circonstances et de l'auditoire: le sens du conte est donc inséparable de sa ré-énonciation et de sa performance, invitant aux improvisations, variations, détours voire détournements.

Les contes littéraires gardent la trace de leurs lointaines origines orales, même si l'histoire du genre montre bien que l'écrit n'est jamais une simple retranscription de la tradition orale mais plutôt sa mise en scène et son imitation souvent parodique. Du Pañcatantra indien au Pentamerone de Giambattista Basile, des Histoires ou contes du temps passé de Charles Perrault aux Kinder- und Hausmärchen des frères Grimm, ces histoires ont traversé les époques, les continents, les langues et les cultures en mêlant des sources très diverses, tant populaires que littéraires ${ }^{1}$. La tradition écrite fait référence à une culture orale associée aux femmes pour mieux s'en distancier, depuis Platon qui recommande de rejeter les contes de nourrices au profit des récits d'Hésiode et d'Homère dans l'éducation des enfants, jusqu'à Perrault qui se réclame de la figure emblématique de "Ma mère l'Oye» représentée sur le frontispice des Histoires ou contes du temps passé (1697), mais de façon assez ambiguë2

I. La distinction entre les Volksmärchen (contes populaires) et les Kunstmärchen (contes littéraires) est apparue à l'époque romantique avec les frères Grimm et l'émergence de la folkoristique comme discipline visant à préserver et valoriser un héritage oral et populaire en voie de disparition. Paul Zumthor invite cependant à ne pas plaquer le couple oral/écrit sur celui de populaire/savant (ou littéraire) dans Le masque et la lumière. A son tour, l'historienne de la culture Marina Warner a documenté les interactions complexes entre des traditions orales, populaires et littéraires dans From the Beast to the Blonde, qui met en évidence comment le conte a été associé aux femmes depuis l'Antiquité. Sur les stratégies de représentation de l'oralité dans les contes littéraires français de la fin du XVII e siècle, voir la préface au volume dirigé par A. Defrance et J.-F. Perrin, Le conte en ses paroles, et notamment l'excellent article de L. Seifert, "Entre l'écrit et l'oral». Jean-François Perrin rappelle que dans le milieu des salons mondains où évolue Perrault, "l'on écrit d'abord pour l'oreille» (Le conte en ses paroles, p. 14), avant que les contes, sous des formes diverses (imprimé, livre illustré, pantomime...), se diffusent auprès de publics toujours plus larges.

2. Voir Le conte en ses paroles, p. 90-103; J. Perrot (dir.), Les métamorphoses du conte, en particulier l'article de M. Manson sur «Platon et les contes de nourrices». 


\section{Charles Perrault et la parole feinte: le clin d'oeil à la tradition orale}

Puisant dans les collections italiennes de Straparola et de Basile (entre autres), la mode du conte de fées qui voit le jour dans les salons parisiens à la fin du XVII e siècle a donné à un genre longtemps méprisé ses lettres de noblesse et sa légitimité littéraire. Comme l'ont bien montré les spécialistes de la culture mondaine à l'époque de Louis XIV, les conteuses et les conteurs de l'Ancien Régime échangent leurs créations dans les salons. Ils mettent en scène ce mode de transmission oral dans le récit-cadre de leurs recueils, les frontispices, les préfaces ou les contes eux-mêmes, et se réclament d'une oralité qui recouvre des formes et des sens divers. Lewis C. Seifert souligne à juste titre que l'«oralité» "peut désigner à la fois une tradition narrative, la voie de transmission de celle-ci, une situation de contage, sinon l'énonciation au niveau de la narration ou de la diégèse. Et si, de surcroît, nous considérons les champs sémantiques que cette oralité peut évoquer par connotation ou par métonymie - l'enfance, le peuple, la culture populaire, et de façon floue, le passé - nous prenons conscience alors de la complexité de cette notion ${ }^{3}$.

Charles Perrault fréquente les salons où les aristocrates pratiquent l'art de la conversation en "mitonnant» des contes, comme Marie-Catherine d'Aulnoy, Charlotte-Rose de Caumont de la Force, Marie-Jeanne L'Héritier de Villandon et Catherine Bernard. Ce divertissement raffiné et galant permet d'exercer son esprit et sa plume sur un thème donné, tout en abordant des sujets controversés tels que l'éducation des filles, le bon ou le mauvais usage de la parole, l'importance des sentiments et les rapports de classe. Le genre naïf et enfantin du conte est ici un "masque» qui permet de manier l'ironie et de formuler une fine critique sociale dans les limites de la bienséance tout en exerçant une relative liberté.

La démarche poétique de Perrault s'inscrit à dessein dans une recherche du «naturel» qui cultive la spontanéité, la naïveté et l'ingénuité attribuées à l'oralité. L'écrivain français marque ainsi son appartenance aux «Modernes», et raille l'esprit de sérieux attribué aux doctes, érudits et pédants imitateurs des «Anciens» dans la fameuse Querelle ${ }^{4}$.

3. L. Seifert, «Entre l'écrit et l'oral», p. 21.

4. Voir les travaux pionniers de Jacques Barchilon, et plus récemment ceux d'Anne Defrance, Marc Escola, Donald Haase, Jean Mainil, Sophie Raynard, Raymonde 
Perrault était en effet un membre éminent de l'Académie et il a occupé, comme son frère, des charges importantes à la cour avant de tomber en disgrâce après la mort de Colbert. Il est indéniable que des contes circulaient dans la culture orale et les classes populaires en Europe, dont les emblématiques "contes de Peau d'Ane» consignés dans la Bibliothèque bleue. Mais ils ne sont pas valorisés comme tels avant l'époque romantique et l'intérêt pour la Volkspoesie 5 . Si Perrault se réclame des «contes de vieilles" et des "contes de nourrices", ce n'est pas parce qu'il est un folkloriste avant la lettre, mais parce que la culture populaire liée à l'enfance et aux femmes devient chez lui l'emblème d'une poétique «moderne» incarnant la culture française: raffinée et élégante, mais plaisante, enjouée et sans prétention. Comme il le déclare dans sa préface aux Contes en vers de 1695, les contes sont des «bagatelles» qui ne sont pas pour autant dépourvues de "Morale». Pour citer Marc Escola à propos des vers de L'Héritier soulignant le plaisir inoffensif procuré par les contes entendus dans l'enfance, que reprend Perrault dans la préface à son propre recueil:

Au fond, sous couvert de "naïveté» et au bénéfice de la simple «douceur», trois traditions se trouvent tout à la fois assumées et subverties: la tradition orale, la tradition satirique, la tradition morale. Et à qui sait être plusieurs lecteurs à la fois, le vers final parle clair: on ne trouvera rien à redire de ces narrations qui redisent le conte oral sans le répéter ${ }^{6}$.

Le conte de nourrice, genre féminin sans prestige ni autorité, se réinvente ainsi dans la reprise, le dédoublement, et la différence joueuse et railleuse mais «sans fiel et sans malignité » (L'Héritier citée par Perrault dans la

Robert, Lewis C. Seifert, Jean-Paul Sermain, Catherine Velay-Vallantin et Marina Warner.

5. Un vieux débat oppose les défenseurs de l'origine populaire des contes aux tenants d'une tradition littéraire et savante comme source de créativité à travers l'histoire. Cette querelle révèle des présupposés méthodologiques et des intérêts disciplinaires spécifiques, mais aussi des positionnements politiques, sociaux, idéologiques et même philosophiques distincts (phonocentrisme vs scriptocentrisme, intérêt pour l'art populaire vs art des élites etc.). Il est aujourd'hui admis que les cultures orales et écrites sont inséparables et complémentaires, et qu'elles s'inspirent l'une de l'autre depuis la plus haute Antiquité.

6. M. Escola, Marc Escola commente, p. 42. 
préface aux Contes, p. 53). De même, la dédicace des contes en prose de Perrault à une princesse invitée à produire sa propre interprétation du texte, et le choix d'un frontispice représentant une «instance d'énonciation orale, celle d'une voix du conte" sont les aspects d'une "stratégie à double face", pour emprunter l'heureuse formule de Louis Marin ${ }^{7}$ (citée par Escola p. 72), dont la portée est à la fois politique, éthique et esthétique. Celui-ci a d'ailleurs observé une modification subtile dans le manuscrit des contes de Perrault, où la référence à ceux qui «écoutent" ces histoires naïves est remplacée par "ceux qui les lisent» dans l'édition imprimée de 1697. Cet effet de doublure et l'injonction faite au lecteur - ici à la lectrice - de chercher un sens "qui se découvre plus ou moins selon le degré de pénétration de ceux qui les lisent» ("A Mademoiselle», Contes, p. 127) sont autant d'invitations à déployer de multiples niveaux de lecture. Selon Jean-François Perrin, la mise en scène de l'oralité dans les contes de Perrault participe ainsi d'une "poétique de la parole et de la voix figurée» ${ }^{8}$. Puisant dans les arts du discours de l'époque, cette poétique prend comme modèle la "simplicité» de la parole vive. Elle joue sur la tonalité et le volume des voix, les styles et les registres de langue (y compris le parler populaire), le rythme et la ponctuation des échanges verbaux (pour dire la menace, la surprise, l'émotion, etc.), ce qui explique sans doute le succès des contes de Perrault auprès d'un large public, leurs adaptations sur la scène de théâtre (théâtre comique, pantomime, etc.), et plus récemment à la radio et au cinéma.

L'oralité figurée dans "Peau d'Ane», "La Belle au bois dormant", "Le Petit chaperon rouge", "Les Souhaits ridicules» et "Le Chat botté"

Sans surprise, la plupart des contes de Perrault traitent du pouvoir de la parole et célèbrent l'art de dire, tout en se proposant de (re)créer un «style oral" par l'écriture. Le conte en vers de "Peau d'Ane", dédié à AnneThérèse de Marguenat de Courcelles, elle-même salonnière et auteure de traités de morale, en donne un bon exemple. Perrault propose de se laisser bercer par les "agréables sornettes" que sont les "contes d'Ogre et de Fée» plutôt que les récits ennuyeux que préfèrent les "Anciens» (Racine,

7. Citée par M. Escola, Marc Escola commente, p. 72.

8. A. Defrance, J.-F. Perrin, Le conte en ses paroles, p. 9. 
Boileau...), admirateurs inconditionnels et imitateurs de la culture antique. Il reprend donc cette histoire emblématique du genre ("conte de Peau d'Ane" est alors synonyme de "conte populaire») et raconte à son tour l'histoire de cette jeune princesse qui parvient à échapper aux visées incestueuses de son père en se dissimulant sous une peau de bête. L'histoire familière (et audacieuse par son thème) se voit "élevée» dans une forme raffinée et versifiée, même si Perrault achève son récit par un retour au souvenir d'enfance et à la scène emblématique du contage féminin "naïf». Le conte de "Peau d'Ane», dit-il, persistera dans les mémoires " tant que dans le Monde on aura des Enfants, / Des Mères et des Mèresgrands» (Contes, p. 115). Perrault fait référence à la scène du frontispice où une femme raconte des histoires à un jeune public rassemblé autour d'elle, qui constitue la «doublure» populaire et féminine de sa propre entreprise littéraire et subtilement parodique. De la même manière, un autre conte en vers drôlatique, "Les Souhaits Ridicules", relate "La folle et peu galante fable» (Contes, p. 119) de Jupiter accordant trois souhaits à un bûcheron et sa femme qui s'empressent de les galvauder. L'épouse se retrouve avec une saucisse suspendue au bout du nez et ne s'en débarrasse qu'au prix du dernier souhait. Le prologue s'adresse à la destinataire du conte, la très aristocratique "Mademoiselle de La $\mathrm{C}^{* * *}$ ", qui elle aussi sait «charmer en racontant, / Et dont l'expression est toujours si naïve, / Que l'on croit voir ce qu'on entend" (Contes, p. 119). Le dernier vers joue sur le double sens du mot "entendre»: l'art de conter "charme» l'auditoire en stimulant l'imagination de celles et ceux qui écoutent, mais il fait aussi appel à l'intelligence et sollicite la capacité interprétative de l'auditoire, puisque "entendre» signifie également "comprendre». Le jeu des rimes fait ainsi "entendre" un rapprochement comique entre "l'Achéron» des anciens et le "bûcheron» des modernes, et les lecteurs avisés sauront y voir un clin d'oeil burlesque à la Querelle. Dans tous les cas, Perrault loue l'effet de présence qui fait tout l'art paradoxal du conteur ou de la conteuse mondaine qui imite la "naïveté», la simplicité, la fantaisie et l'humour des contes populaires, mais en y insufflant un esprit de cour qui évoque la reine Marie-Antoinette jouant à la bergère.

Les contes en prose de Perrault déplacent les prologues ou épilogues des contes en vers, mêlant galanterie et auto-référentialité ludique, dans des moralités versifiées. La dimension orale du conte est intégrée au récit lui-même, qui devient le lieu où l'auteur témoigne de son rapport distancié à la tradition populaire. Le conte littéraire permet-il à son tour 
de "faire voir» ce que l'on dit ou ce que l'on lit? Tel est l'enjeu d'un conte comme "Les Fées», où les paroles d'une jeune fille serviable et aimable se transforment en perles et diamants lorsqu'elle raconte "naïvement" (Contes, p. 166) à sa mère sa rencontre avec une fée, alors que les paroles de sa méchante sœur se changent en vipères et crapauds. Marie-Jeanne L'Héritier, nièce de Perrault, a elle aussi proposé une version de cette histoire dans «Les Enchantements de l'éloquence ou les effets de la douceur", dont le titre programmatique résume bien la poétique du conte merveilleux comme art de la parole et éthique de la civilité.

Le célèbre conte de "La Belle au bois dormant» thématise lui aussi les pouvoirs de la parole, qui déploie toute son efficacité performative quand les sept fées marraines se penchent sur le berceau de la princesse et lui accordent divers dons, dont celui de chanter "comme un Rossignol" (Contes, p. 132). Ces dons sont aussitôt suivis de la malédiction de la vieille fée («elle dit [...] que la princesse se percerait la main d'un fuseau, et qu'elle en mourrait" p. 132), atténuée par une jeune fée prévoyante cachée derrière la tapisserie, "qui dit tout haut ces paroles: "Rassurezvous, Roi et Reine, votre fille n'en mourra pas. [...] Elle tombera seulement dans un profond sommeil qui durera cent ans, au bout desquel un fils de Roi viendra la réveiller.” "(p. 132). La malédiction se réalise malgré les précautions du roi, car la parole des fées est "reine» si l'on peut dire. Cent ans plus tard, un prince aperçoit les tours du château par delà la forêt infranchissable. Le pouvoir de la parole est réaffirmé quand le prince s'enquiert de l'histoire du lieu. Cette fois-ci, il ne s'agit plus des puissantes fées de cour, mais de simples gens du peuple: «chacun lui répondit selon qu'il en avait ouï parler. Les uns disaient que c'était un vieux Château où il revenait des Esprits, les autres que tous les Sorciers de la contrée y faisaient leur sabbat. La plus commune opinion était qu'un Ogre y demeurait, et que là il emportait tous les enfants qu'il pouvait attraper, pour pouvoir les manger à son aise.» (p. 134). C'est le récit d'un vieux paysan qui se souvient d'une histoire entendue "il y a plus de cinquante ans" qui provoque l'effet le plus spectaculaire sur le jeune prince: "Le jeune Prince, à ce discours, se sentit tout de feu" (p. 135). Il s'identifie aussitôt au héros capable de réveiller la belle princesse endormie, et l'histoire racontée par le paysan a suffi à donner au jeune homme le goût de l'aventure romanesque et à tomber amoureux de la jeune fille à sa seule évocation. Comme les cent ans sont révolus, le prince franchit la forêt sans difficulté et trouve la princesse endormie; 
elle se réveille spontanément (il n’y est pour rien), le baiser «romantique» n'apparaissant que bien plus tard chez les Grimm. La prédiction des fées ne peut se réaliser qu'avec la collaboration d'un simple paysan, dont le récit fait advenir la romance improbable?

La scène de la rencontre galante met en scène une princesse pleine d'esprit (le narrateur soulignant avec malice qu'elle a eu beaucoup de temps pour préparer sa répartie) et un prince un peu empoté qui mâ̂trise mal l'art de la conversation: "Ses discours furent mal rangés, ils en plurent advantage; peu d'éloquence, beaucoup d'amour» (p. 136). La condition amoureuse transcende heureusement ces maladresses, et donne lieu à un échange verbal nourri («il y avait quatre heures qu’ils se parlaient...", p. 136). Le reste est vite expédié: repas et cérémonie religieuse font place à une longue nuit de noces sans sommeil (les jeunes époux ayant semble-t-il encore beaucoup de choses tendres à se dire, comme le souligne le narrateur indiscret et malicieux). La deuxième partie du conte fait résonner très différemment le sens du mot «tendre». La mère du prince est en effet une ogresse qui, en l'absence de son fils, cherche à dévorer la princesse et ses deux enfants. Ces derniers échappent de justessse à la voracité de leur mère-grand grâce à un subterfuge du maître d'hôtel qui prend pitié de la princesse, de la petite Aurore et du petit Jour. On notera que la monstruosité de la belle-mère ogresse est "audible" plutôt que visible, comme dans cet échange avec le cuisinier: "Je veux manger à mon dîner la petite Aurore. - Ah! Madame, dit le Maître d'Hôtel. - Je le veux, dit la Reine (et elle le dit d'un ton d'Ogresse qui a envie de manger de la chair fraîche), et je la veux manger à la Sauce-robert» (p. 137-8). La comédie macabre qui se joue ici, entre raffinement gastronomique et cruauté proverbiale des ogres, déploie tous ses effets piquants (comme la sauce) dans la mise en scène des voix des personnages du conte, et dans le décalage entre le récit des événements et le commentaire "naïf» du narrateur ${ }^{10}$.

Le conte du "Petit Chaperon Rouge» avertit pour sa part des dangers de la voix déguisée et de la parole trompeuse. Ce conte célèbre entre tous est composé de plusieurs dialogues entrecoupés de brèves séquences

9. Voir M. Hennard Dutheil de la Rochère, V. Dasen (éds), Des Fata aux fées, et en particulier l'article «Kiss and Tell» de D. Haase.

Io. On note aussi que la reine et ses enfants sont trahis par leur voix: "L'Ogresse reconnut la voix de la Reine et de ses enfants...» (Ch. Perrault, Contes, p. 139). 
narratives. L'oralité y est figurée par les paroles échangées entre l'enfant et sa mère, l'enfant et le loup dans la forêt, le loup et la grand-mère, et enfin le loup et le petit chaperon rouge. Même le bruit des coups frappés à la porte sont transcrits par une onomatopée: «Il heurte: Toc, toc. "Qui est là?" - C'est votre fille le petit chaperon rouge (dit le Loup, en contrefaisant sa voix)»(p. 144). La scène se répète avec le petit chaperon rouge "qui entendit la grosse voix du Loup, [et] eut peur d'abord, mais croyant que sa Mère-grand était enrhumée, répondit [...]»(p. 144). Le Loup s'efforce alors d'adoucir sa voix grave: l'enfant ne reconnaît pas celle de sa grand-mère, mais trouve une raison logique à son altération. Ayant rejoint le Loup dans le lit, elle s'étonne alors de la métamorphose physique de sa mère-grand dans le célèbre échange final fait de reprises et d'échos, avant d'être dévorée dans un dénouement brutal et dramatique: " "C'est pour te manger." Et en disant ces mots, ce méchant Loup se jeta sur le petit chaperon rouge, et la mangea» (p. 145). Perrault semble même avoir ajouté une indication de lecture incitant le conteur à joindre le geste à la parole (à l'instar du loup du conte!) en se jetant sur l'enfant qui écoute, même si cette note manuscrite est sans doute apocryphe. La morale du conte enjoint les jeunes filles à ne pas «écouter toute sorte de gens», surtout si elles sont «belles, bien faites, et gentilles» (p. 145). Car le "Loup" de Perrault emprunte à la fable: la morale fait "entendre" que les "Loups doucereux" sont des libertins qui exercent leur art de la séduction par la parole, et le conte souligne le danger de se laisser «conter fleurette».

«Le Maître Chat ou le Chat Botté» présente lui aussi de nombreux dialogues où le "Chat" fabulateur intimide et manipule ses interlocuteurs à sa guise. De fait, la morale du conte ne peut être qu'ironique. Dès le début du conte, le chat répond à son maître, qui menace de le manger, avec aplomb et sang-froid, et il expose "d'un air posé et sérieux" (p. 157) la première mesure à adopter pour les sortir tous les deux de la misère. C'est parce qu'il est autorisé à parler au roi qu'il parvient à le convaincre de l'existence du fictif, puissant et richissime "Marquis de Carabas» - car, nous dit le narrateur dans une parenthèse qui souligne toute la fantasie de l'animal, "c'était le nom qu'il lui prit en gré de donner à son Maître» (p. 158). Plus tard, il fait répéter aux paysans son propre mensonge en les menaçant de représailles ("vous serez tous hachés menu comme chair à pâté», p. 159), et joue enfin magistralement de la flatterie avec un ogre vaniteux doué du pouvoir de se métamorphoser 
avant d'arriver à ses fins. Après avoir feint (?) d'être effrayé par l'ogre changé en lion, il le met au défi de se changer en souris en jouant sur la réputation de ce nouveau Protée:

On m’a assuré encore, dit le Chat, mais je ne saurais le croire, que vous aviez aussi le pouvoir de prendre la forme des plus petits Animaux $[\ldots] »($ p. 160).

Joignant le geste à la parole, l'ogre se transforme en souris ... et il est aussitôt dévoré par le chat. Ainsi, la parole produit des effets fabuleux et permet de changer l'ordre des choses en transformant un miséreux fils de meunier en beau-fils de roi grâce aux ruses et à la faconde de son chat.

\section{Angela Carter, ou la radio au service de la tradition orale}

A la suite de Simone de Beauvoir dans Le deuxième sexe (1949), de nombreuses féministes ont dénoncé l'influence pernicieuse sur les jeunes filles des contes glorifiant la beauté et la passivité féminine dès leur plus jeune âge. L'écrivaine anglaise Angela Carter (1940-1992) a pris part à ce débat en soulignant pour sa part l'infinie capacité de métamorphose des histoires familières, tant formelle que structurelle, stylistique, sémantique ou générique. Ce qu'elle a brillamment illustré à travers sa démarche de "transcréation " et de "transmédiation» des contes, qui reflète un projet esthétique d'une grande audace, inventivité, originalité et liberté, mais aussi l'engagement politique de l'auteure en faveur de l'émancipation des femmes et la mise en évidence de leur contribution à l'histoire culturelle et littéraire. Carter donne une nouvelle actualité aux contes de Perrault dans The Fairy Tales of Charles Perrault (1977). Ce projet de traduction et les recherches qu'elle entreprend sur le genre deviennent le matériau à partir duquel elle élabore ses propres variations sur les contes dans plusieurs genres et mediums. Suite à sa traduction pour les enfants, Carter revisite les "vieilles histoires" pour des lecteurs adultes. Rassemblées dans The Bloody Chamber and Other Stories (1979), ces réécritures en prose du "Petit Chaperon rouge», de "La Belle au Bois dormant» et du "Chat Botté» (entre autres) sont désormais célèbres. Les versions radiophoniques de ces réécritures, en revanche, sont beaucoup moins connues. Elles s'élaborent pourtant en contre-point des nouvelles, Carter mettant cette fois-ci en évidence l'héritage oral et populaire 
du conte. Alors que les nouvelles s'attachent à la dimension textuelle et intertextuelle des contes conformément au projet de l'auteure selon lequel l'émancipation des femmes passe par la relecture critique et créative des textes du passé, la radio permet de valoriser l'art du contage comme le nouveau lieu de "la magie " ${ }^{11}$. Cette démarche fait écho à un regain d'intérêt pour l'oralité comme forme d'expression pivilégiée de la culture populaire et pour l'héritage méconnu du conte féminin qui allait faire l'objet de plusieurs études dans les sciences humaines et sociales à partir des années 1960.

Plusieurs nouvelles parues dans The Bloody Chamber ont ainsi leur "doublure» radiophonique dans les radio plays réalisés par Angela Carter entre 1976 et 1984, réunis dans Come Unto These Yellow Sands: Four Radio Plays (1985). Carter collaborera ensuite avec le cinéaste Neil Jordan sur le scénario du film The Company of Wolves (1984), qui s'inspire de plusieurs réécritures du "Petit Chaperon Rouge" réunies dans The Bloody Chamber. Les pièces radiophoniques reprennent trois contes qui présentent une forte dimension orale et jouent sur les voix: "Le Petit Chaperon Rouge» dans The Company of Wolves, "La Belle au Bois dormant» dans Vampirella, et "Le Chat Botté» dans Puss in Boots. Carter explore les potentialités thématiques, structurelles et formelles qui se révèlent lorsque l'histoire est transposée dans un nouveau medium. La réécriture en prose et la pièce radiophonique servent à explorer la subjectivité et le désir féminins à partir de diverses stratégies narratives, stylistiques, métatextuelles et intertextuelles d'une part, et la dimension orale des contes à travers les voix et la musique, ainsi que l'utilisation d'effets spéciaux (bibliothèque sonore) d'autre part. Plus tard, l'adaptation cinématographique va donner lieu à des expérimentations de plus en plus audacieuses dans le cadre de nouveaux projets collaboratifs. Carter réalisera notamment un documentaire controversé pour la télévision, "The Holy Family Album» (1991), un livret d'opéra inspiré de Virginia Woolf,

II. Voir M. Hennard Dutheil de la Rochère, Reading, Translating, Rewriting. Sur l'imaginaire des langues étrangères et le jeu sur leurs sonorités chez Carter, voir aussi M. Hennard Dutheil de la Rochère (éd.), Angela Carter traductrice - Angela Carter en traduction, p. 1-12. On note que l'intérêt de Carter pour l'oralité s'étend au théâtre de Shakespeare qui joue un rôle central dans son dernier roman, Wise Children (1991). Dans un entretien accordé à John Bailey quelques mois avant sa mort, Carter déclare: "One of the things that I've deeply respected about Shakespeare was that [...] he was actually writing for the voice» (http://www.bbc.co.uk/archive/writers/12245.shtml). 
"Orlando: The Enigma of the Sexes", et d'autres projets restés inachevés qui témoignent de son intérêt pour l'intermédialité.

Dans ses pièces radiophoniques, Carter cherche à renouveler le sens du conte pour des auditeurs et auditrices modernes qui, comme elle, sont des "enfants de la radio", comme elle le dit dans la préface à Come Unto These Yellow Sands ("Born in 1940, I was a child of the Radio Age», p. 8). L'idée est de diffuser les contes à travers ce medium populaire et démocratique par excellence, en accord avec l'engagement social et politique de l'auteure. Carter cherche ainsi à reproduire l'expérience "archaïque» associée au genre par le biais des technologies modernes qui permettent de retrouver "la magie des voix dans la nuit» ${ }^{12}$. Elle évoque dans la préface à Come Unto These Yellow Sands l'expérience universelle et intemporelle que représente le fait d'écouter une histoire, et questionne la valorisation de l'écrit et de la vision dans la culture occidentale:

For me, writing for radio involves a kind of three-dimensional storytelling. Anyone, anywhere, who sits down to tell a story, from the narrator of fabulous epics in a pre-literate African community to a travelling salesman embarking on an anecdote in a bar, does so without the help of visual aids [...]. Yet, as with all forms of story-telling that are composed in words, not in visual images, radio always leaves that magical and enigmatic margin, the space of the invisible, which must be filled in by the imagination of the listener ${ }^{13}$.

I2. A. Carter, Come Unto Those Yellow Sands, p. 13. L'intérêt de Carter pour la technologie et l'influence des media de masse sur les modes de perception (et donc sur l'éducation) se retrouve dans les travaux de M. McLuhan. The Gutenberg Galaxy postule que la culture occidentale a passé par plusieurs stades en sollicitant d'abord la vue, puis l'ouïe (qui caractérise l'époque moderne selon lui) et enfin le système nerveux central par la télévision, le cinéma, et les media électroniques. La célèbre formule de McLuhan "The medium is the message» a peut-être joué un rôle dans la démarche de transcréation de Carter. Je remercie Jean-Pierre Bobillot d'avoir attiré mon attention sur ce lien. L'archive de Vampirella (Carter Papers, MS 88899/1/47, British Library) contient des notes et plusieurs versions préparatoires tapuscrites annotées qui montrent comment Carter élabore la nouvelle "The Lady of the House of Love» à partir de la pièce radiophonique ("Change of emphasis - concentrate on erotic relation between Hero and Countess").

13. A. Carter, Come Unto Those Yellow Sands, p. 7. «Pour moi, écrire pour la radio implique une sorte de narration tridimensionnelle. N'importe qui, n'importe où, celui ou celle qui s'assied pour raconter une histoire, du narrateur d'épopées fabuleuses dans une communauté africaine pré-littéraire au vendeur itinérant qui se lance dans une anecdote de bar, le fait sans l'aide de supports visuels [...]. Cependant, comme pour 
La radio permet ainsi de faire revivre un art qui échappe au biais «masculin» du mode visuel vivement critiqué à cette époque, et le medium sollicite l'imagination des auditeurs en adéquation avec la conception active et participative de la création prônée par l'auteure:

It is the necessary open-endedness of the medium, the way the listener is invited into the narrative to contribute to it his or her own way of «seeing" the voices and the sounds, the invisible beings and events, that gives radio story-telling its real third dimension, which is the space that, above all, interests and enchants me ${ }^{14}$.

Ses expérimentations ultérieures avec le medium cinématographique s'attacheront plutôt à la dimension iconique du Petit Chaperon Rouge filtrée à travers la culture populaire. Comme l'a bien montré Michelle Ryan-Sautour dans son article sur les trajectoires intermédiales de ce conte dans l'œuvre de Carter, la transposition à l'écran permet à l'écrivain et au cinéaste de travailler sur le "choc» des images et des couleurs (avec le rouge comme fil... rouge), de réaliser des métamorphoses spectaculaires grâce aux effets spéciaux, et de multiplier les points de vues sur cette histoire familière par le décadrage et la mise en abyme, dans un film d'horreur "postmoderne» qui suscite une réflexion sur la politique de genre du régime visuel.

Carter termine la préface à Come Unto these Yellow Sands en insistant sur l'importance que revêt pour elle son travail pour la radio dans sa démarche d'écrivain, chaque adaptation constituant une oeuvre à part entière, tout en étant liée à un projet plus vaste qui explore les possibilités de chaque medium:

Indeed, radio remains a challenging medium, because so much is possible in it. I write for radio by choice, as an extension and

toutes les formes de narration composées de mots et non d'images visuelles, la radio laisse toujours cette marge de magie et d'énigme, l'espace de l'invisible, qui doit être rempli par l'imagination de l'auditeur» (les traductions de Carter sont de Julie Sîrbu, révisées par l'auteure de l'article).

I4. A. Carter, Come Unto Those Yellow Sands, p. 7. «C'est l'adaptabilité nécessaire du medium, cette façon d'inviter l'auditeur à contribuer à la narration avec sa propre manière de "voir" les voix et les sons, les êtres et événements invisibles, qui donne à la narration radiophonique sa véritable troisième dimension, et c'est cet espace qui m'intéresse et m'enchante par-dessus tout». 
an amplification of writing for the printed page; in its most essential sense,even if stripped of all the devices of radio illusion, radio retains the atavistic lure, the atavistic power, of voices in the dark, and the writer who gives the words to these voices retains some of the authority of the most antique tellers of tales ${ }^{15}$.

Le travail effectué par Carter sur la dimension orale des contes est mis en évidence dans la pièce radiophonique intitulée Vampirella. A la différence des deux autres "radio plays", Vampirella a été d'abord imaginé par Carter pour la radio. Je m’attacherai en particulier à la façon dont les voix humaines sont agencées comme une partition musicale à travers la diction particulière, le rythme et l'accent des personnages à la manière d'une sonate de chambre. Par ailleurs, cette version gothico-parodique du conte de "La Belle au bois dormant» va durablement influencer le tour «sombre» pris par les adaptations de contes contemporaines, au cinéma comme ailleurs ${ }^{16}$.

Du corps du texte aux voix dans la nuit: Vampirella, ou comment échapper à la voix de son maître

Comme d'autres féministes de la "deuxième vague", Carter se souciait de la dimension politique et idéologique de la culture visuelle et de ses implications pour les femmes, à la suite des travaux de John Berger (Ways of Seeing, BBC series, 1972) et de Laura Mulvey sur le regard masculin dans Visual Pleasure and Narrative Cinema (écrit en 1973 et publié en 1975 dans la célèbre revue de théorie du cinéma Screen) ${ }^{17}$. Cette

15. A. Carter, Come Unto Those Yellow Sands, p. 13. "En effet, la radio reste un medium qui représente un défi, parce qu'il rend possible tant de choses. J'écris pour la radio par choix, pour prolonger et amplifier les écrits imprimés sur la page; dans son sens le plus essentiel, même lorsqu'elle est dénuée de tout dispositif d'illusion radiophonique, la radio conserve l'attrait atavique, le pouvoir archaïque des voix dans l'obscurité, et l'écrivain qui met des mots dans ces voix retrouve quelque chose de l'autorité des conteurs les plus anciens».

I6. Voir l'ouvrage pionnier de Ch. Crofts, “Anagrams of Desire” Angela Carter's Writing for Radio, Film and Television.

17. Laura Mulvey connaissait Angela Carter, comme elle me l'a confirmé lors d'un récent colloque à l'Université de Lausanne (Scopophilia, 4-5 juin 2015). 
recherche d'une alternative à la culture visuelle se double chez Carter d'un intérêt pour la culture populaire, la musique et les traditions orales. Cherchant à recréer la voix du conte dans un contexte contemporain, Carter écrit et réalise son premier "radio play", Vampirella, diffusé par Radio 4 le 20 juillet 1976, rediffusé le 29 mai 1977 sur Radio 3, et très récemment sur Radio $4^{18}$.

Carter explique dans la préface à Come Unto These Yellow Sands comment cette pièce radiophonique est née du son «métallique et presque musical» de son crayon d'écrivain courant sur un radiateur dans un moment de rêverie ${ }^{19}$. Ce son évoque des ongles longs et pointus glissant le long des barreaux d'une cage; aiguisés comme les serres d'un oiseau de proie, ce sont ceux de la Comtesse Vampirella qui séduit les hommes et les tue d'un baiser mortel avant de se gorger de leur sang. L'auteur procède par allitération pour recréer le personnage de "La Belle au bois dormant", issue des sonorités liquides de la langue (lovely, lady, sleeping, last, line, locked, castle...):

I alliterated her. A lovely lady vampire. [...] A lovely lady vampire; last of her line, perhaps, locked up in her hereditary Transylvanian castle $[\ldots]^{20}$.

A partir de cette genèse musicale lointainement inspirée du conte de Perrault, c'est le son des mots qui guide Carter dans l'élaboration du récit. Plus précisément, l'auteure met en évidence ce qui, dans le conte qu'elle traduit à la même époque, ressortit spécifiquement au medium radiophonique et à la sonorité des mots anglais (un "syndrome" thématisé dans le vampirisme qui affecte son héroïne dotée d’une bouche sensuelle et monstrueuse) :

I8. http://www.bbc.co.uk/programmes/b06tfv03 et http://www.bbc.co.uk/ programmes/b01by8n1

19. "But I started writing for radio, myself, because of a sound effect. I made it quite by accident. Sitting in my room, pencil in hand, staring vacantly into space instead of getting on with whatever it was I was supposed to be doing, I ran the pencil idly along the top of the radiator. It made a metallic, almost musical rattle. It was just the noise that a long, pointed, fingernail might make if it were run along the bars of a birdcage» (A. Carter, Come Unto Those Yellow Sands, p. 9).

20. Ibid., p. 9. "Je l'ai allitérée. Une jolie lady vampire. [...] Une jolie lady vampire, dernière de sa lignée, peut-être, enfermée dans son château héréditaire de Transylvanie $[\ldots] »$. 
I never thought of any other medium but radio all the time I was writing the script of the play, Vampirella. It came to me as radio, with all its images formed, in terms of words and sounds ${ }^{21}$.

La dimension dramatique du conte se prête évidemment à l'adaptation radiophonique, mais pour Carter la radio offre surtout un espace privilégié pour créer des récits complexes "en trois dimensions" qui permettent d'entrecroiser les récits des divers protagonistes et leurs temporalités propres:

This is, just, the way I like to use radio, not for creating dramas on a theatrical model so much as to create complex, many-layered narratives that play tricks with time ${ }^{22}$.

Carter va puiser les effets sonores dans les archives de la BBC avec la complicité de la productrice Glyn Dearman, et jouer sur la riche gamme d'intonations de la voix humaine ("the human voice itself imparts all manner of subtleties in its intonations ", p. 10), du murmure amoureux au cri d'agonie, en passant par les soupirs, sanglots, gémissements, rires et hurlements. Davantage que l'écrit, la matière sonore des mots et le grain de la voix humaine dans ses infinies modulations deviennent des instruments qui stimulent la mémoire sensorielle des auditeurs, et rendent sensibles les ambiguïtés du conte:

The rich textures of radio are capable of stating ambiguities with a dexterity over and above that of the printed word ${ }^{23}$.

Carter joue en particulier sur l'ambiguïté générique du conte de Perrault, qui hésite entre la comédie noire et le pathos bizarre, selon ses propres termes. Elle compose ainsi autour de la figure de la Comtesse Vampirella, en proie aux remords et à un spleen baudelairien, une gallerie de portraits sonores de personnages à la fois grotesques et monstrueux associés à des

2I. A. Carter, Come Unto Those Yellow Sands, p. 10. "Je n'ai jamais pensé à un autre medium que la radio en écrivant Vampirella. Elle m'est venue en tant que pièce radiophonique, avec toutes ses images déjà prêtes, sous la forme de mots et de sons».

22. Ibid., p. 7. "C'est ainsi seulement que j'aime utiliser la radio, pas tant pour créer des drames sur un modèle théâtral que pour créer des récits complexes à couches multiples qui permettent de jouer avec la temporalité».

23. Ibid., p. 10. «Les riches textures de la radio permettent d'exprimer l'ambiguïté bien plus habilement que l'écrit». 
identités linguistiques marquées, comme les anthropophages Mr. et Mrs Beane, dont l'accent écossais s'accorde avec le son de la cornemuse, ou le nécrophile parisien Henri Blot avec son fort accent français. Mais surtout, le conte produit une autre figure familière par homophonie en français (comme Carter l'avait sans doute noté en traduisant les contes de Perrault) : celle du comte Dracula avec son accent des Carpathes. Le personnage de Dracula évoque l'ogresse monstrueuse de Perrault et son goût immodéré pour la chair fraîche à la Sauce Robert. Il est aussi une créature littéraire anglo-saxonne par excellence (issue du Dracula de Bram Stoker en 1897) et cinématographique (Dracula de Tod Browning, 1931) dont l'acteur fétiche, Béla Lugosi, était célèbre autant par son physique d'outre-tombe que pour son inimitable diction mêlant l'anglais aux accents hongrois. Vampirella a pour sa part une prédilection très aristocratique pour le français, alors que la population environnante parle une langue "full of Ks and Ts" qui bruit autour du Héros anglais égaré dans les Carpathes (p. 88). La pièce radiophonique témoigne de la fascination de l'auteure pour les variétés linguistiques, la gamme des accents et des registres, les inflections, tonalités et modulations de la langue qui révèlent l'origine sociale et géographique, mais aussi le caractère et la nature des personnages. L'activité de traduction fait entendre la gamme des sonorités et invite à jouer sur les mots, y compris entre les langues, comme matrice de création. De nombreuses voix, langues et langages se bousculent ainsi dans l'anglais de Carter, qui a trouvé dans leurs sonorités particulières la possibilité de nouvelles histoires, et fait du mélange des sons et des sangs la source vive de sa créativité.

Transmission, répétition, variation, subversion: "Can a bird sing only the song it knows, or can it learn a new song?"

La pièce radiophonique s'ouvre d'ailleurs sur le personnage principal, Vampirella, qui s'interroge sur la possibilité d'échapper à la fatalité et à la répétition du même par la reprise du motif du chant d'oiseau, empruntée au conte de Perrault, qui devient ici un refrain mélodique à la dimension métafictionnelle évidente. On s'en souvient, les fées qui se penchent sur le berceau de la Belle au bois dormant lui accordent de nombreux dons, et en particulier celui de chanter "comme un Rossignol", avant que la vieille fée fâchée de ne pas avoir été conviée à la fête condamne la 
petite princesse à mourir jeune, une malédiction transmuée en sommeil de cent ans.

Carter s'empare du motif sonore du rossignol pour en déployer les résonances à la fois littéraires et musicales en anglais à travers les célèbres poèmes romantiques de Percy Bysse Shelley, "To a Skylark», et celui de John Keats, "Ode to a Nightinghale», où le poète addresse son poignant appel à l'oiseau invisible ("My heart aches, and a drowsy numbness pains [...].») Chez Carter, le chant mélodieux de l'oiseau est interrompu par un cri de chauve-souris, qui fait basculer la poésie lyrique dans l'univers gothique, et la mélodie dans le mélo. Le rire musical de la Comtesse est repris par des notes de harpe qui soulignent la proximité de la voix humaine et de l'instrument de musique: "The Countess laughs: and her laughter is picked up by a harp that mirrors her laughter» (p. 84). Carter souligne par divers procédés l'imitation du réel par l'artifice (comme le conte imite l'oralité) et joue avec dextérité et humour sur le pouvoir évocateur des bruits caractéristiques de l'univers gothique: outre la diction artificielle et le style affecté de son héroïne, le grincement sinistre qui marque l'ouverture du tombeau de Dracula, les hoquets de stupeur et d'effroi des spectateurs qui découvrent le vampire gisant dans la tombe, et la voix caverneuse du Comte recréent tout un monde imaginaire par la magie des effets sonores.

Les soupirs appuyés de la Comtesse mélancolique, ses accents mélodramatiques et sa prose scandée d'artistocrate contribuent à porter l'attention de l'auditeur sur le rythme, la cadence et les sonorités de la voix humaine comme musique:

COUNTESS: I am the lady of the castle. My name is exile. My name is anguish. My name is longing. Far, far from the world on the windy crest of the mountain, I am kept in absolute seclusion, my time passes in endless revery, a perpetual swooning. I am both the Sleeping Beauty and the enchanted castle; the princess drowses in the castle of her flesh ${ }^{24}$.

24. A. Carter, Come Unto Those Yellow Sands, p. 90. "Je suis la dame du château. Mon nom est exil. Mon nom est angoisse. Mon nom est désir. Loin, très loin de ce monde sur la crête venteuse de la montagne, je suis retenue dans un isolement absolu, mon temps s'écoule dans une rêverie sans fin, une pâmoison perpétuelle. Je suis à la fois la Belle au bois dormant et le château enchanté; la princesse somnole dans le château de sa chair". 
Cette figure qui vit dans la nuit est à la fois un leurre et la formule symbolique (a symbolic formula, p. 93) qui donne le ton de la pièce radiophonique.

Vampirella disparaît lorsque le jeune touriste anglais dont elle est tombée amoureuse l'embrasse innocemment sur le front pour lui souhaiter bonne nuit, et par ce geste (qui fait écho à celui des parents de la jeune princesse endormie dans le conte de Perrault) la condamne à mort - et le récit de vampire avec elle. Ce changement inattendu dans l'intrigue précipite la chute de la maison Dracula et tout son théâtre sonore. Le père de Vampirella ne s'y trompe pas. Il s'écroule dans un cri, suivi d'un râle prolongé: "COUNT. ... aaargh...» (p. 114). Le conte de fée comme genre pour les enfants reprend ses droits, et la romance ( $\mathrm{I}$ always knew that love, true love, would kill me», p. 114) vient mettre un terme au grandguignol vampirique. Du moins jusqu'à la prochaine fois. Le chant matinal de l'alouette est interrompu par le son du tambour militaire, et le Comte a le dernier mot, glaçant, en voix off, en nous renvoyant à la réalité sanglante du monde:

COUNT: The shadow of the Fatal Count rises over every bloody battlefield.

Everywhere, I am struck down; everywhere, I celebrate my perennial resurrection ${ }^{25}$.

Le travail expérimental de Carter sur le conte traverse les frontières entre les genres, les arts et les media (radio, télévision, film). Il met aussi en évidence l'intérêt manifeste de l'auteur pour le travail collaboratif avec les responsables du programme de la $\mathrm{BBC}$ et l'équipe de techniciens et d'acteurs impliqués dans la production de pièces radiophoniques. Cette démarche de «transcréation" et de "transmédiation" met délibérément en questions les hiérarchies entre les genres et rend manifeste le goût de Carter pour les langues étrangères comme façon de moduler sa propre langue et d'en rendre sensible la matière sonore. Ainsi, Carter a souhaité faire revivre une tradition orale archaïque et universelle par la «magie» des technologies modernes. Mais la réflexion qu'elle élabore sur le merveilleux technologique a aussi sa part sombre et tragique, symbolisée

25. A. Carter, Come Unto Those Yellow Sands, p. 116. «L'ombre du Comte Fatal s'élève au-dessus de chaque champ de bataille sanglant. Partout, je suis abattu; partout, je célèbre ma résurrection à jamais recommencée». 
par la première guerre mondiale - première guerre industrielle marquée par une véritable "technique de la mort». La musique du monde (le chant d'oiseau à l'aube) s'interrompt à la fin de Vampirella, quand le bric-à-brac gothique incarné par Vampirella fait place au spectre de Dracula qui hante les tranchées. La véritable horreur n'est pas dans la fiction (conte d'ogre ou récit de vampire) mais bel et bien dans le réel et l'Histoire ("avec sa grande hache», pour citer Georges Perec) où la guerre fait couler le sang et tomber les morts.

Martine Hennard Dutheil de la Rochère Université de Lausanne 


\section{BIBLIOGRAPHIE}

BBC Radio 4: "Writing in Three Dimensions: Angela Carter's Love Affair with Radio»(I6.02.2002) (http://www.bbc.co.uk/ programmes/b01by8n1).

Carter, Angela, Come Unto Those Yellow Sands: Four Radio Plays, (s. 1.) [London], Bloodaxe Books Ltd., 1985.

-, The Bloody Chamber and Other Stories, London, Vintage, 2006 (1979).

Crofts, Charlotte, "Anagrams of Desire" Angela Carter's Writing for Radio, Film and Television, (s. 1.) [Manchester], Manchester University Press, 2012.

Defrance, Anne, Perrin, Jean-François, Le conte en ses paroles. La figuration de l'oralité dans le conte merveilleux du Classicisme aux Lumières, Paris, Desjonquères, coll. «L'esprit des Lettres», 2007.

Escola, Marc, Marc Escola commente: contes de Charles Perrault, Paris, Folio, 2005.

Haase, Donald, "Kiss and Tell: Orality, Narrative, and the Power of Words in "Sleeping Beauty" ", in Martine Hennard Dutheil de la Rochère, Véronique Dasen (éds), Des Fata aux fées: regards croisés de l'Antiquité à nos jours, Etudes de Lettres, 289 (2011/3-4), p. 279296.

Hennard Dutheil de la Rochère, Martine, Reading, Translating, Rewriting: Angela Carter's Translational Poetics, Detroit, Wayne State University Press, 2013.

—, «La magie des mots étrangers: lire, traduire et réécrire (chez) Angela Carter", in Martine Hennard Dutheil de la Rochère (éd.), Angela Carter traductrice - Angela Carter en traduction, Lausanne, Cahiers du CTL, 2014, p. 11-27.

Hennard Dutheil de la Rochère, Martine, Dasen, Véronique (éds), Des Fata aux fées: regards croisés de l'Antiquité à nos jours, Etudes de Lettres, 289 (2011/3-4). 
Manson, Michel, "Platon et les contes de nourrices", in Jean Perrot (éd.), Les métamorphoses du conte, Bruxelles, Peter Lang, 2004, p. 27-40.

Mcluhan, Marshall, The Gutenberg Galaxy: The Making of Typographic Man, Toronto, University of Toronto Press, 1962.

ONG, Walter, Orality and Literacy: The Technologizing of the Word, Londres et New York, Routledge, 1982.

Perrault, Charles, Contes, éd. par Jean-Pierre Collinet, (s. 1.) [Paris], Folio, 1981.

Perrot, Jean (dir.), Les métamorphoses du conte, Paris, Seuil jeunesse, 1998.

Ryan-Sautour, Michelle, "The Intermedial Trajectories of Angela Carter's Wolf Tales", Journal of the Short Story in English, 59, (2012), p. 75-91 (http://jsse.revues.org/1322).

Seifert, Lewis C., «Entre l'écrit et l'oral: la réception des contes de fées "classiques" " in Anne Defrance, Jean-François Perrin, (éds), Le conte en ses paroles. La figuration de l'oralité dans le conte merveilleux du Classicisme aux Lumières, Paris, Desjonquères, 2007, p. 21-33.

Velay-Vallantin, Catherine, "Charles Perrault, la conteuse et la fabuliste: "l'image dans le tapis"", Féeries, 7 (2010), p. 95-121.

Warner, Marina, From the Beast to the Blonde: On Fairy Tales and their Tellers, New York, Farrar, Straus et Giroux, 1996.

—, «Marina Warner on why Angela Carter's The Bloody Chamber Still Bites", The Scotsman, 15 September 2012 (www.scotsman.com/ lifestyle/culture/books/marina-warner-on-why-angela-carter-sthe-bloody-chamber-still-bites-1-2528708).

Zumthor, Paul, Le masque et la lumière, Paris, Seuil, 1978. 